\title{
Laurence Moon Bardet Biedl Syndrome with Maturity Onset Diabetes of the Young (Mody) - Case Report
}

\author{
Krishna Kumar Dhakchinamoorthi ${ }^{1, *}$, John Thomas Palathingal ${ }^{1}$, Saroja Sreenivasan ${ }^{2}$ \\ 'Departemt of Pharmacy Practice, C.L. Baid Metha College of Pharmacy, Chennai, Tamil Nadu, INDIA. \\ 2Department of Diabetology, Voluntary Health Services Hospital, Adyar, Chennai, Tamil Nadu, INDIA.
}

\begin{abstract}
Laurence-Moon-Bardet-Biedl syndrome (LMBBS) is an inherited genetic condition combination of two distinct disorders: Biedl-Bardet syndrome (BBS) and Laurence-Moon syndrome (LMS). BBS is characterized by polydactyly fingers or toes, obesity, renal failures and learning difficulties. The clinical signs of this condition vary among affected person, most person with Bardet-Biedl syndrome also develop a blurred central vision. LMS is a rare condition that may include cerebellar ataxia; vision abnormalities due to peripheral neuropathy; spastic paraplegia and intellectual disability. The cardinal clinical features include retinitis pigmentosa, obesity, polydactyly and hypogenitalism. The clinical complications significantly vary among differently affected siblings, include obesity, type 2 diabetes, elevated blood pressure and elevated cholesterol levels (hypercholesterolemia), renal complications, hypopituitarism; and short stature. This case study is
\end{abstract}

first to report maturity-onset diabetes of the young (MODY) in LaurenceMoon-Bardet-Biedl syndrome among two south Indian patients.

Key words: Laurence-Moon-Bardet-Biedl Syndrome, MODY, Diabetes.

Correspondence

Dr. Krishna Kumar Dhakchinamoorthi, M.Pharm., Ph.D, Professor and Head Department of Pharmacy Practice, C.L. Baid Metha College of Pharmacy, Chennai, INDIA.

Phone: +919894465615

Email: krishnakumarrx@gmail.com

DOI: 10.5530/jyp.2019.11.25

\section{INTRODUCTION}

Laurence-Moon-Bardet-Biedl (LMBB) syndrome is an autosomal recessive disorder characterized by retinitis pigmentosa, polydactyly, obesity, mental retardation, hypogenitalism, renal dysplasia and short stature..$^{1,2}$ Many individuals with Bardet-Biedl syndrome also have reported with renal abnormalities, which can be serious or life-threatening. ${ }^{3}$ Other clinical features of Bardet-Biedl syndrome reported such as impaired speech, delayed development of motor skills, behavioral abnormalities such as immatured emotional and inappropriate outbursts and poor coordination. Other distinctive features are facial and dental abnormalities, unusual short or fused fingers or toes and loss of the sense of smell have been reported in some cases with Bardet-Biedl syndrome. ${ }^{4}$ LaurenceMoon syndrome (LMS) was later termed Laurence-Moon-Bardet-Biedl syndrome because of similarities with Bardet-Biedl syndrome (BBS). There are several disagreements due to differences in the underlying pathogenetic causes of these syndromes. However, these two syndromes reported to have similar features to Oliver-McFarlane syndrome (OMS). All three syndromes are characterized by progressive blindness, obesity and learning disabilities. ${ }^{4}$ Maturity-onset diabetes of the young (MODY) is a heterogeneous inherited genetic disorder that results in $\beta$-cell dysfunction characterized by non-insulin dependent form of diabetes. It occurs in adolescence or young adults before the age of 25 years. ${ }^{5}$ Type II diabetes reported in patients with LMBB Syndrome, however not reported distinct as MODY. ${ }^{6}$ The present case report examined two siblings (a male and a female) born of consanguineous marriage suffering from a genetic disorder with marked similar clinical manifestations.

\section{Case Report}

Case 1: A 26 years old female patient admitted for amputation of fifth toe and an extra toe on the right leg with the complaints of diabetic foot ulcer. The patient was presented with a history of 10 years of type II diabetes mellitus, nyctalopia (in the past 2 years), optic atrophy, atypical retinopathy with maculopathy and obesity. Also presented with hexadactyly, she was the first child of a consanguineous marriage. She initially developed night blindness (nyctalopia) at the age of 2 years and later developed optic atrophy and atypical retinopathy with maculopathy at the age of 20 years. She had multiple hospital admissions for diabetes management. Initially treated as type I diabetes and later diagnosed as MODY (Maturity-onset diabetes of the young). Later during the phase of the management, the patient became obese and had a delay in the menstrual cycles. She is $150 \mathrm{~cm}$ tall and weighed $92 \mathrm{~kg}$ with a body mass index (BMI) of $39.3 \mathrm{~kg} / \mathrm{m}^{2}$. She had a dim vision, had marked central obesity, a round face, brachycephaly and postaxial polydactyly (24 fingers; one removed during childhood; two toes removed via amputation) (Figure 1 and 2).

Case 2: A 24 years old male patient visited in the diabetology ward with the history of 8 years of type II diabetes mellitus which was initially treated as type I diabetes mellitus (MODY). He had a problem of nyctalopia from 2 years of age which later developed into optic atrophy and retinitis pigmentosa. He is $152 \mathrm{~cm}$ tall and body weight of $94 \mathrm{~kg}$ with a BMI of $39.9 \mathrm{~kg} / \mathrm{m}^{2}$. He had a gradual increase in body weight during the management of diabetes mellitus which later became consistent. He had a dim vision, central obesity, a round face, brachycephaly and postaxial polydactyl ( 24 fingers). The patient's penis and testis were small and hair over the genitalia was sparse. (Figure 3 and 4).

\section{INVESTIGATIONS}

Routine investigations showed elevated sugar levels with fluctuation during insulin therapy (Figure 5 and 6), While lipid profile, renal and liver function tests were normal. Urine analysis revealed $420 \mathrm{mg} / 1500 \mathrm{ml} /$ day

This is an open access article distributed under the terms of the Creative Commons Attribution-NonCommercial-ShareAlike 4.0 License, which allows others to remix, tweak, and build upon the work non-commercially, as long as the author is credited and the new creations are licensed under the identical terms. 


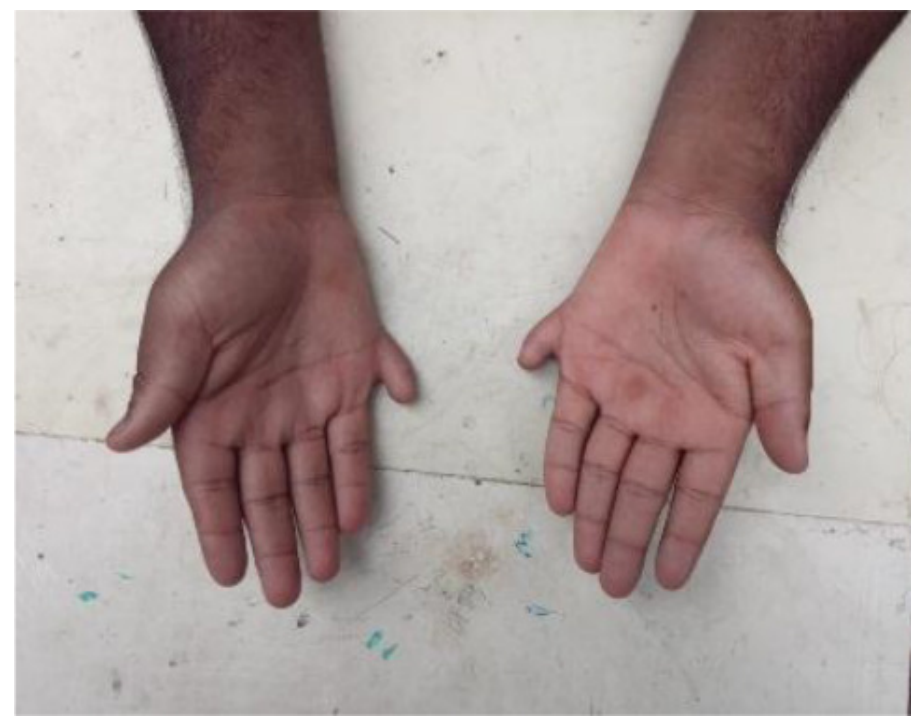

Figure 1: Female sibling had Postaxial polydactyl fingers in her hands.

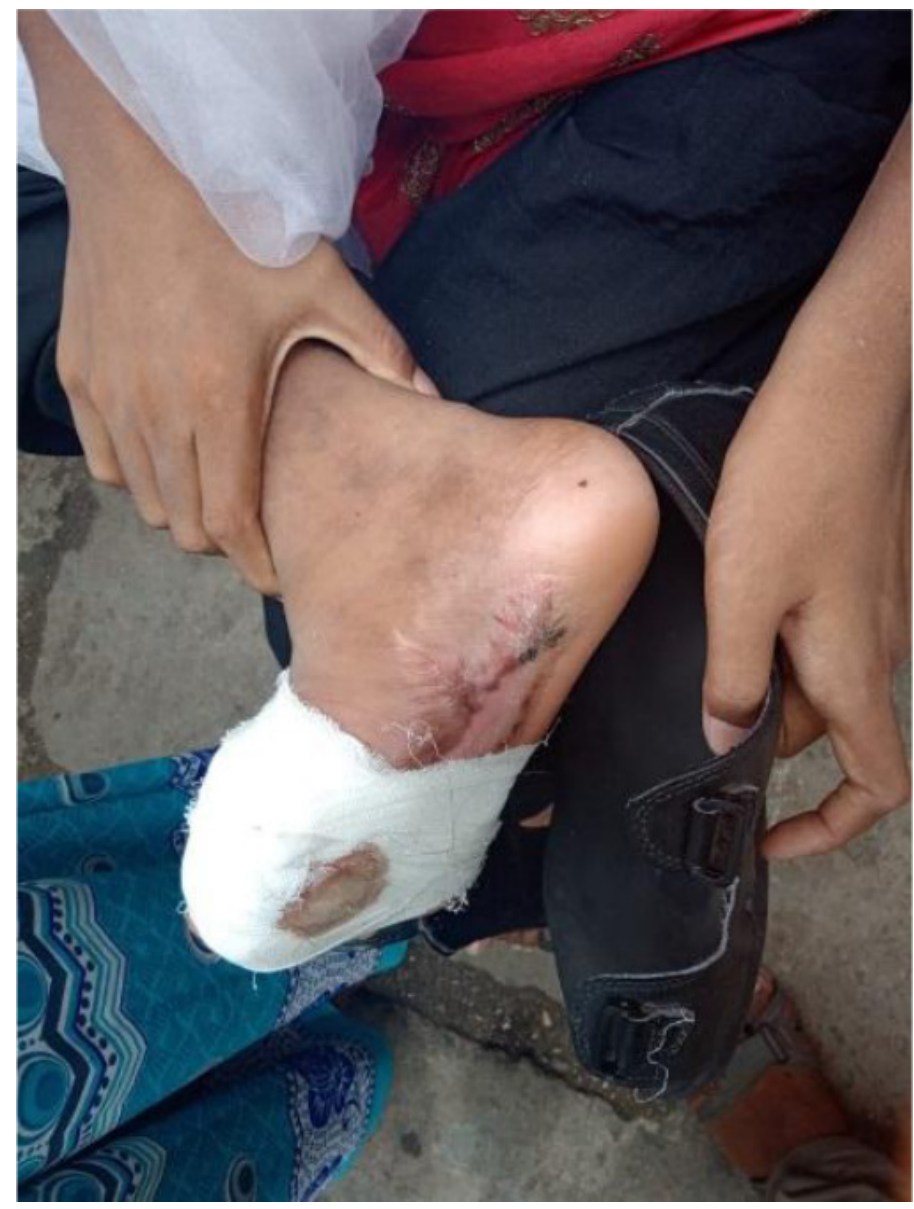

Figure 2: Female sibling had Postaxial polydactyl fingers in the legs and toes removed via amputation.

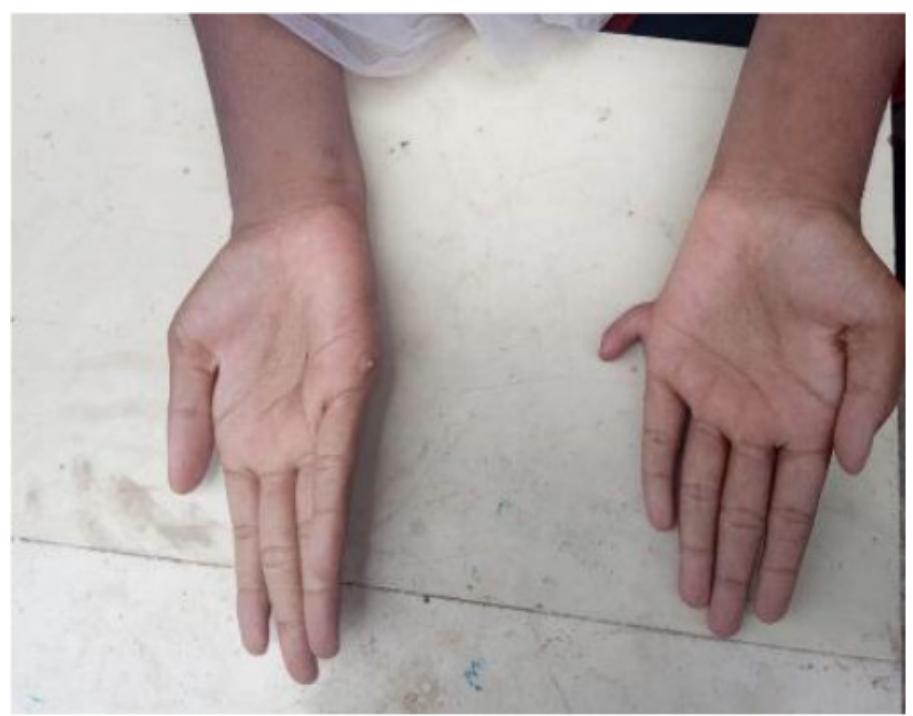

Figure 3: Male sibling had Postaxial polydactyl fingers in his hands.

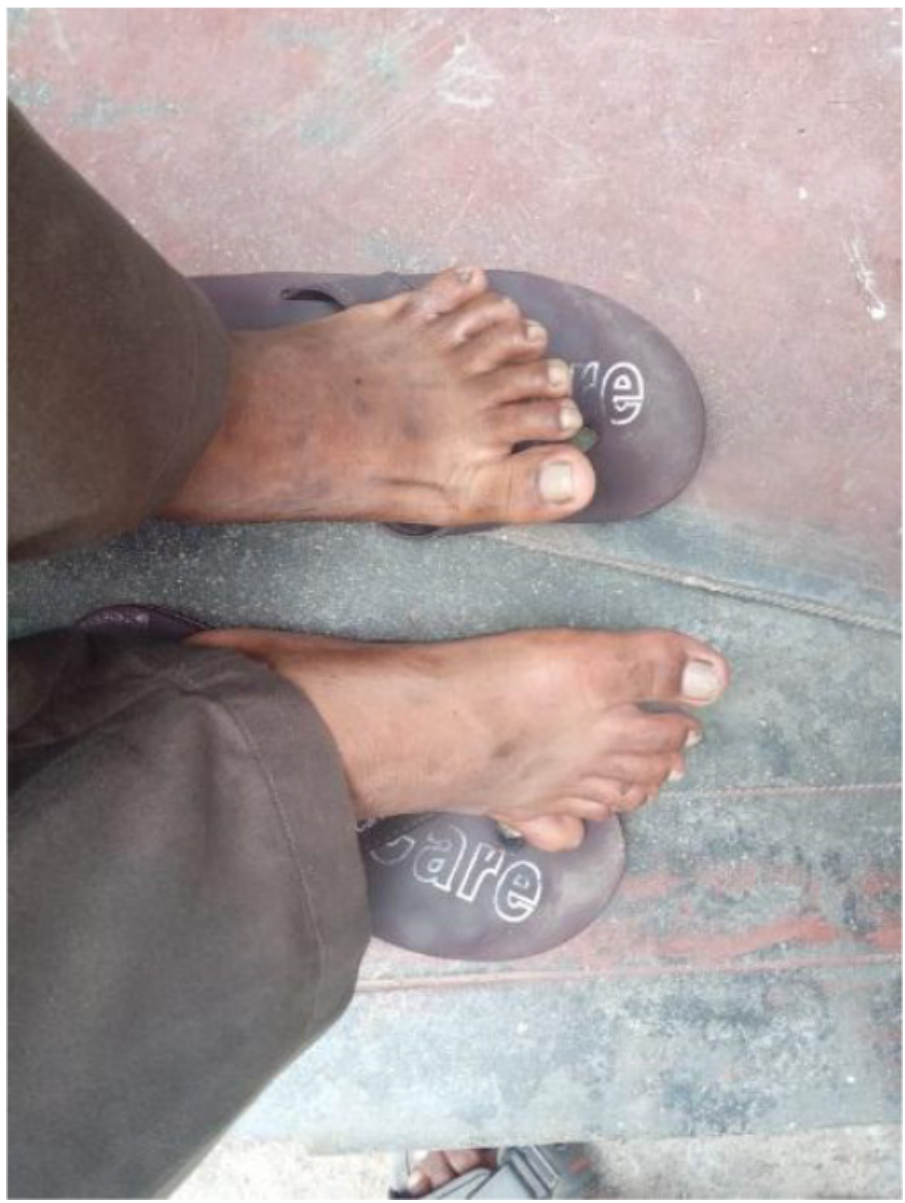

Figure 4: Male sibling had Postaxial polydactyl fingers in the legs. 


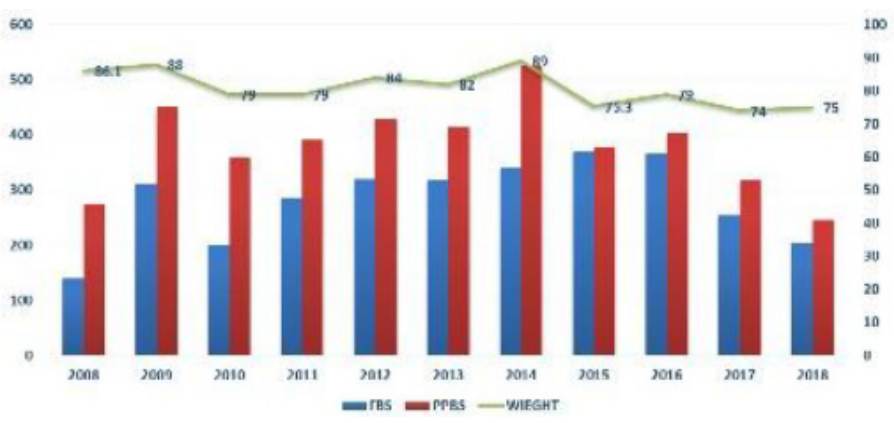

Figure 5: Ten years fluctuation of blood sugar with body weight in female sibling.

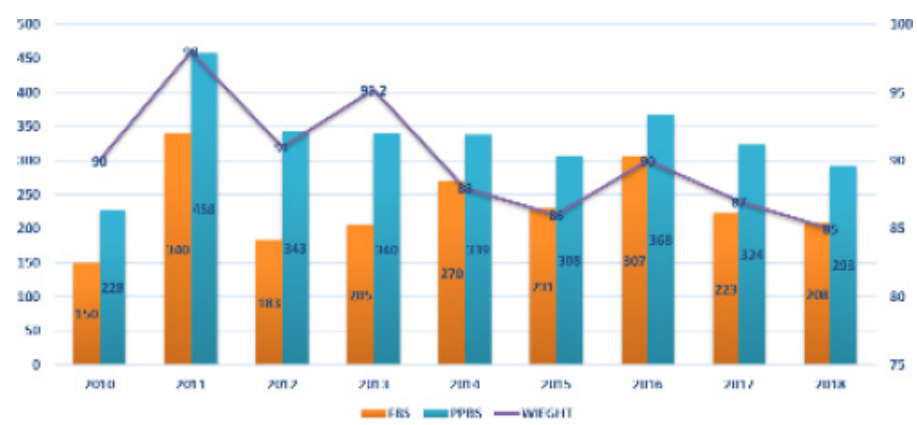

Figure 6: Eight years fluctuation of blood sugar with body weight in male sibling.

of proteinuria. Ultrasonography revealed normal renal anatomy. Fundoscopy reveals retinitis pigmentosa.

\section{TREATMENT}

Initially considering the elevated sugar levels, the patient was administered with insulin and gradually it was changed into OHA (oral hypoglycemic agents) Metformin $500 \mathrm{mg}$, (Two) doses in the morning and one at night), Glimepiride $2 \mathrm{mg}$ (at morning and night) and again shifted back to insulin. The second male case is treated also with voglibose $0.2 \mathrm{mg}$ (thrice a day). Routine ophthalmic examinations were done. The management of LMBB syndrome requires supportive care and was provided after consulting an occupational therapist, psychologist and social worker. Management of obesity was achieved through diet and lifestyle modification. To improve the outcome in vision there is no effective treatment available in BBS.

\section{DISCUSSION}

The prevalence of Bardet-Biedl syndrome (BBS) disorder varies based on geographical location. The exact pathogenesis of BBS is unknown, however, a variant $B B S$ gene $(B B S 1-B B S 15)$ involved in ciliary function reported as to cause BBS. ${ }^{1}$ In our case report, two siblings were diagnosed with Bardet-Biedl syndrome having the following clinical features such as type II diabetes mellitus, hexadactyl, nyctalopia, optic atrophy, atypical retinopathy with maculopathy and obesity. These two siblings were children of the consanguineous marriage. In a previous case study reported similar clinical features also in agreement that there is an increased occurrence of Bardet-Biedl syndrome in consanguineous marriages. ${ }^{7}$ In the present cases patients were found to have polydactyly, hypogonadism and retinitis pigmentosa which was earlier reported in south Indian siblings who had the similar clinical features proving that consanguineous marriage is one of the risk factors for BBS. ${ }^{8}$ However, contrary to our report there is a case study on 17-year-old patient with clinical manifestations of night blindness, obesity and polydactyly, but was not a child of consanguineous marriage. ${ }^{2}$ Similarly, another case report on BBS in a 35-year-old patient reported with most of the clinical features but not from a consanguineous marriage. ${ }^{9}$ The Prevalence of MODY in Indian population was reported to be higher (North India (20\%) with symptomatic hyperglycemia) than the Caucasians (4.8\%). MODY reported as a rare cause of diabetes ( $1 \%-2 \%$ among all diabetes). However, it is frequently misdiagnosed as type I or type II diabetes. ${ }^{10}$ In a previous case report thirteen years male patient reported to had Laurence -Moon-Bardet- Biedl syndrome with Diabetes Mellitus, however they have not reported distinct as MODY form of diabetes. In the present case report with clear clinical features and regular monitoring, we reported the MODY in Laurence -Moon-Bardet- Biedl syndrome.

\section{CONCLUSION}

Laurence Moon Bardet Biedl syndrome with maturity-onset diabetes of the young (MODY) is a genetically and clinically heterogeneous group of conditions. In both the genetically inherited condition, consanguineous marriages were seen to be the main risk factor. Diagnosis remains a challenge for clinicians, knowledge of these rare causes of diabetes and BBS with additional clinical findings and other genetic biomarkers will hopefully improve its detection rates.

\section{ACKNOWLEDGEMENT}

Mr. Dan Monsy, Ms.Binitha Ann Thomas and Mr.Christy John Daniel PharmD students are gratefully acknowledged.

\section{CONFLICT OF INTEREST}

The authors declare that there is no conflict of interest.

\section{ABBREVIATIONS}

BBS: Bardet-Biedl syndrome; MODY: Maturity-Onset Diabetes of the Young; OMS: Oliver-McFarlane syndrome; OHA: Oral hypoglycemic agents.

\section{REFERENCES}

1. Forsythe E, Beales PL. Bardet-Biedl syndrome. Eur J Hum Genet. 2013;21(1):8-13.

2. Laurence JZ, Moon RC. Four cases of 'retinitis pigmentosa' occurring in the same family and accompanied by general imperfections of development. Obes Res. 1995;3(4):400-3.

3. Singh KK, Kumar R, Prakash J, Krishna A. Bardet-Biedl syndrome presenting with steroid-sensitive nephrotic syndrome. Indian J Nephrol. 2015;25(5):300-2

4. Laurence-Moon Syndrome. Genetic and Rare Diseases Information Center. Last updated: 6/18/2015. https://rarediseases.info.nih.gov/diseases/12635/ laurence-moon-syndrome.

5. Bisht R. Maturity Onset Diabetes of the Young (MODY) - An essence. J Med Res. 2016;2(2):24-5

6. Quader M, Islam MS, Ahmed QMU, Azad MAK, Rahim MA. Laurence -MoonBardet- Biedl syndrome with Diabetes Mellitus. J Med. 2012;13(1):97-9.

7. Madireddi J, Acharya V, Suryanarayana J, Hande H, Shetty R. Bardet-Biedl syndrome: multiple fingers with multiple defects. BMJ Case Reports. 2015.

8. Sulaiman S, Basha S, Kaladhar G, Reddy S. Laurence Moon Bardet Biedl Syndrome-A Case Report. Asian Pac J Health Sci. 2016;3(4):209-11.

9. Chakravarti H, Ray S, Chakrabarti S, Biswas D, Ghosh S, Mukhopadhyay S, et al. Bardet-Biedl syndrome in two siblings: a rare entity revisited. QJM-Int J Med. 2015;109(2):123-4

10. Gardner DSL, Tai ES. Clinical features and treatment of maturity-onset diabetes of the young (MODY). Diabetes Metab Syndr Obes. 2012;5:101-8. 